\title{
Agent-Based Negotiation in Uncertain Environments
}

\author{
John Debenham and Carles Sierra
}

\begin{abstract}
An agent aims to secure his projected needs by attempting to build a set of (business) relationships with other agents. A relationship is built by exchanging private information, and is characterised by its intimacy - degree of closeness - and balance - degree of fairness. Each argumentative interaction between two agents then has two goals: to satisfy some immediate need, and to do so in a way that develops the relationship in a desired direction. An agent's desire to develop each relationship in a particular way then places constraints on the argumentative utterances. The form of negotiation described is argumentative interaction constrained by a desire to develop such relationships.
\end{abstract}

\section{Introduction}

This paper is in the area labelled: information-based agency [10]. An informationbased agent has an identity, values, needs, plans and strategies all of which are expressed using a fixed ontology in probabilistic logic for internal representation and in an illocutionary language [9] for communication. All of the forgoing is represented in the agent's deliberative machinery. We assume that such an agent resides in a electronic institution [1] and is aware of the prevailing norms and interaction protocols.

[9] describes a rhetorical argumentation framework that supports argumentative negotiation. It does this by taking into account: the relative information gain of a new utterance, the relative semantic distance between an utterance and the dialogue history. Then [11] considered the affect that argumentative dialogue has on the ongoing relationship between a pair of negotiating agents. Neither of these contribu-

John Debenham

University of Technology, Sydney, Australia, e-mail: debenham@it.uts.edu.au and Carles Sierra Institut d'Investigació en Intel-ligència Artificial - IIIA, Spanish Scientific Research Council, CSIC,08193 Bellaterra, Catalonia, Spain, e-mail: sierra@iiia.csic.es 
tions addressed the relationship between argumentative utterances or strategies for argumentation.

This paper is based in rhetorical argumentation [8] — we attempt to edge our approach towards classical argumentation by modelling the criteria that the partner uses to gauge the effect of argumentation. For example, suppose I am shopping for a new car and have cited "suitability for a family" as a criterion. The salesman says "This LandMonster is great value.", and I reply "My grandmother could not climb into that." Classical argumentation may attempt to refute the matriarch's lack of gymnastic prowess or the car's inaccessibility. Taking a less confrontational and more constructively persuasive view we might note that this statement impacts negatively on the "suitability for a family" criterion, and attempt to counter that impact possibly with "Its been voted No 1 for children.". Although a smarter response may look for an argument that is semantically closer: "The car's height ensures a very comfortable ride over rough terrain that is popular with old people."

Our argumentation agent has to perform two key functions: to understand incoming utterances and to generate responses. In Section 2 we describe the communication model and an argumentation language that admits Prolog-like statements. The approach is founded on a model of contract acceptance that is described in Section 3. Section 4 details a scenario that provides the context for the discussion. Sections 5 and 6 consider the scenario from each side of the bargaining table. Reactive and proactive argumentation strategies are given in Section 7, and Section 8 concludes.

\section{Communication Model}

This paper is written from the point of view of an agent $\alpha$ that is engaged in argumentative interaction with agent $\beta$. The history of all argumentative exchanges is the agents' relationship. We assume that their utterances, $u$, can be organised into distinct dialogues, $\Psi^{t}$. For simplicity we assume that at most one dialogue exists at any time. We assume that $\alpha$ and $\beta$ are negotiating with the mutual aim of signing a contract, where the contract will be an instantiation of the mutually-understood object $o\left(\Psi^{t}\right)$. We assume that this negotiation is taking place through the exchange of proposals accompanied by argumentative dialogue.

In order to define a language to structure agent dialogues we need an ontology that includes a (minimum) repertoire of elements: a set of concepts organised in a is-a hierarchy (e.g. platypus is a mammal, Australian-dollar is a currency), and a set of relations over these concepts (e.g. price(beer,AUD) [4]:

An ontology is a tuple $\mathscr{O}=(V, R, \leq, \sigma)$ where:

1. $V$ is a finite set of concept symbols (including basic data types), i.e. a vocabulary;

2. $R$ is a finite set of relation symbols;

3. $\leq$ is a reflexive, transitive and anti-symmetric relation on $V$ (a partial order), and

4. $\sigma: R \rightarrow V^{+}$is the function assigning to each relation symbol its arity 
where $\leq$ is the traditional $i s-a$ hierarchy. To simplify computations in the computing of probability distributions we will assume that there is a number of disjoint is-a trees covering different ontological spaces (e.g. a tree for types of fabric, a tree for shapes of clothing, and so on). $R$ contains relations between the concepts in the hierarchy, this is needed to define deals as tuples of issues. Semantic distance plays a fundamental role in strategies for information-based agency, see [10] for details.

The general argumentation language described here was first reported in [11]. The discussion is from the point of view of an information-based agent $\alpha$ in a multiagent system where $\alpha$ interacts with negotiating agents, $\beta_{i}$, and information providing agents, $\theta_{j}:\left\{\alpha, \beta_{1}, \ldots, \beta_{o}, \theta_{1}, \ldots, \theta_{t}\right\}$.

The shape of the language that $\alpha$ uses to represent the information received and the content of its dialogues depends on two fundamental actions: (i) passing information, and (ii) exchanging proposals and contracts. A contract $(a, b)$ between agents $\alpha$ and $\beta$ is a pair where $a$ and $b$ represent the actions that agents $\alpha$ and $\beta$ are responsible for respectively. Contracts signed by agents and information passed by agents, are similar to norms in the sense that they oblige agents to behave in a particular way, so as to satisfy the conditions of the contract, or to make the world consistent with the information passed. Contracts and Information can thus be thought of as normative statements that restrict an agent's behaviour.

$\alpha$ 's communication language has two fundamental primitives: $\operatorname{Commit}(\alpha, \beta, \varphi)$ to represent, in $\varphi$, the world that $\alpha$ aims at bringing about and that $\beta$ has the right to verify, complain about or claim compensation for any deviations from, and Done $(u)$ to represent the event that a certain action $u^{1}$ has taken place. In this way, norms, contracts, and information chunks will be represented as instances of Commit(.) where $\alpha$ and $\beta$ are individual agents. Language $\mathscr{L}$ is the set of utterances $u$ defined as:

$$
\begin{gathered}
u::=\text { illoc }(\alpha, \beta, \varphi, t)|u ; u| \text { Let } \text { context } \mathbf{I n} u \text { End } \\
\varphi::=\text { term }|\operatorname{Done}(u)| \operatorname{Commit}(\alpha, \beta, \varphi)|\varphi \wedge \varphi| \\
\varphi \vee \varphi|\neg \varphi| \forall x . \varphi_{x} \mid \exists x . \varphi_{x} \\
\text { context }::=\varphi \mid \text { id }=\varphi \mid \text { prolog_clause } \mid \text { context; context }
\end{gathered}
$$

where $\varphi_{x}$ is a formula with free variable $x$, illoc is any appropriate set of illocutionary particles, ';' means sequencing, and context represents either previous agreements, previous illocutions, the ontological working context, that is a projection of the ontological trees that represent the focus of the conversation, or code that aligns the ontological differences between the speakers needed to interpret an (illocutionary) action $u$. Representing an ontology as a set predicates in Prolog is simple. The set term contains instances of the ontology concepts and relations. ${ }^{2}$

\footnotetext{
${ }^{1}$ Without loss of generality we will assume that all actions are dialogical.

${ }^{2}$ We assume the convention that $V(v)$ means that $v$ is an instance of concept $V$ and $r\left(v_{1}, \ldots, v_{n}\right)$ implicitly determines that $v_{i}$ is an instance of the concept in the $i$-th position of the relation $r$.
} 
John Debenham and Carles Sierra

\section{Contract Acceptance}

No matter what interaction strategy an agent uses, and no matter whether the communication language is that of simple bargaining or rich argumentation, a negotiation agent will have to decide whether or not to sign each contract on the table. We will argue in Section 5 that the buyer will be uncertain of his preferences in our Scenario described in Section 4. If an agent's preferences are uncertain then it may not make sense to link the agent's criterion for contract acceptance to a strategy that aims to optimise its utility. Instead, we pose the more general question: "how certain am I that $\delta=(\phi, \varphi)$ is a good contract to sign?" - under realistic conditions this may be easy to estimate. $\mathbb{P}^{t}(\operatorname{sign}(\alpha, \beta, \chi, \delta))$ estimates the certainty, expressed as a probability, that $\alpha$ should $\operatorname{sign}^{3}$ proposal $\delta$ in satisfaction of her need $\chi$, where in $(\phi, \varphi) \phi$ is $\alpha$ 's commitment and $\varphi$ is $\beta$ 's. $\alpha$ will accept $\delta$ if: $\mathbb{P}^{t}(\operatorname{sign}(\alpha, \beta, \chi, \delta))>c$, for some level of certainty $c$.

To estimate $\mathbb{P}^{t}(\operatorname{sign}(\alpha, \beta, \chi, \delta)), \alpha$ will be concerned about what will occur if contract $\delta$ is signed. If agent $\alpha$ receives a commitment from $\beta, \alpha$ will be interested in any variation between $\beta$ 's commitment, $\varphi$, and what is actually observed, as the enactment, $\varphi^{\prime}$. We denote the relationship between commitment and enactment:

$$
\mathbb{P}^{t}\left(\operatorname{Observe}\left(\alpha, \varphi^{\prime}\right) \mid \operatorname{Commit}(\beta, \alpha, \varphi)\right)
$$

simply as $\mathbb{P}^{t}\left(\varphi^{\prime} \mid \varphi\right) \in \mathscr{M}^{t}$, and now $\alpha$ has to estimate her belief in the acceptability of each possible outcome $\delta^{\prime}=\left(\phi^{\prime}, \varphi^{\prime}\right)$. Let $\mathbb{P}^{t}\left(\operatorname{acc}\left(\alpha, \chi, \delta^{\prime}\right)\right)$ denote $\alpha$ 's estimate of her belief that the outcome $\delta^{\prime}$ will be acceptable in satisfaction of her need $\chi$, then we have:

$$
\mathbb{P}^{t}(\operatorname{sign}(\alpha, \beta, \chi, \delta))=f\left(\mathbb{P}^{t}\left(\boldsymbol{\delta}^{\prime} \mid \delta\right), \mathbb{P}^{t}\left(\operatorname{acc}\left(\alpha, \chi, \delta^{\prime}\right)\right)\right)
$$

for some function $f ;{ }^{4}$ if $f$ is the arithmetic product then this expression is mathematical expectation. $f$ may be more sensitive; for example, it may be defined to ensure that no contract is signed if there is a significant probability for a catastrophic outcome.

There is no prescriptive way in which $\alpha$ should define $\mathbb{P}^{t}\left(\operatorname{acc}\left(\alpha, \chi, \delta^{\prime}\right)\right)$, it is a matter for applied artificial intelligence to capture the essence of what matters in the application. In any real application the following three components at least will be required. $\mathbb{P}^{t}\left(\operatorname{satisfy}\left(\alpha, \chi, \delta^{\prime}\right)\right)$ represents $\alpha$ 's belief that enactment $\delta^{\prime}$ will satisfy her need $\chi . \mathbb{P}^{t}\left(\operatorname{obj}\left(\delta^{\prime}\right)\right)$ represents $\alpha$ 's belief that $\delta^{\prime}$ is a fair deal against the open marketplace - it represents $\alpha$ 's objective valuation. $\mathbb{P}^{t}\left(\operatorname{sub}\left(\alpha, \chi, \delta^{\prime}\right)\right)$ represents $\alpha$ 's belief that $\delta^{\prime}$ is acceptable in her own terms taking account of her ability to meet her commitment $\phi$ [9] [10], and any way in which $\delta^{\prime}$ has value to her personally ${ }^{5}$ — it represents $\alpha$ 's subjective valuation. That is:

\footnotetext{
${ }^{3}$ A richer formulation is $\mathbb{P}^{t}\left(\operatorname{eval}(\alpha, \beta, \chi, \delta)=e_{i}\right)$ where eval $(\cdot)$ is a function whose range is some descriptive evaluation space containing terms such as "unattractive in the long term".

${ }^{4} \beta$ influences the equation in the sense that different $\beta$ s yield different $\mathbb{P}^{t}\left(\delta^{\prime} \mid \delta\right)$.

${ }^{5}$ For example, when buying a new digital camera, $\alpha$ may give a high subjective valuation to a camera that uses the same memory cards as her existing camera.
} 
Agent-Based Negotiation in Uncertain Environments

$$
\mathbb{P}^{t}\left(\operatorname{acc}\left(\alpha, \chi, \delta^{\prime}\right)\right)=g\left(\mathbb{P}^{t}\left(\operatorname{satisfy}\left(\alpha, \chi, \delta^{\prime}\right)\right), \mathbb{P}^{t}\left(\operatorname{obj}\left(\delta^{\prime}\right)\right), \mathbb{P}^{t}\left(\operatorname{sub}\left(\alpha, \chi, \delta^{\prime}\right)\right)\right)
$$

for some function $g$.

Suppose that an agent is able to estimate: $\mathbb{P}^{t}\left(\operatorname{satisfy}\left(\alpha, \chi, \delta^{\prime}\right)\right), \mathbb{P}^{t}\left(\operatorname{obj}\left(\delta^{\prime}\right)\right)$ and $\mathbb{P}^{t}\left(\operatorname{sub}\left(\alpha, \chi, \delta^{\prime}\right)\right)$. The specification of the aggregating $g$ function will then be a strictly subjective decision. A highly cautious agent may choose to define:

$$
\mathbb{P}^{t}\left(\operatorname{acc}\left(\alpha, \chi, \delta^{\prime}\right)\right)= \begin{cases}1 & \text { if: } \mathbb{P}^{t}\left(\operatorname{satisfy}\left(\alpha, \chi, \delta^{\prime}\right)\right)>\eta_{1} \\ & \wedge \mathbb{P}^{t}\left(\operatorname{obj}\left(\delta^{\prime}\right)\right)>\eta_{2} \wedge \mathbb{P}^{t}\left(\operatorname{sub}\left(\alpha, \chi, \delta^{\prime}\right)\right)>\eta_{3} \\ 0 & \text { otherwise }\end{cases}
$$

for some threshold constants $\eta_{i}$. Whereas an agent that was prepared to permit some propagation of confidence from one factor to compensate another could define:

$$
\mathbb{P}^{t}\left(\operatorname{acc}\left(\alpha, \chi, \delta^{\prime}\right)\right)=\mathbb{P}^{t}\left(\operatorname{satisfy}\left(\alpha, \chi, \delta^{\prime}\right)\right)^{\eta_{1}} \times \mathbb{P}^{t}\left(\operatorname{obj}\left(\delta^{\prime}\right)\right)^{\eta_{2}} \times \mathbb{P}^{t}\left(\operatorname{sub}\left(\alpha, \chi, \delta^{\prime}\right)\right)^{\eta_{3}}
$$

where the $\eta_{i}$ balance the influence of each factor.

The point of this is: if an agent aims to produce persuasive argumentative dialogue then in the absence of any specific information concerning the structure of $g$ the agent should ignore $g$ and concentrate on the three categories: $\mathbb{P}^{t}\left(\operatorname{satisfy}\left(\alpha, \chi, \delta^{\prime}\right)\right)$, $\mathbb{P}^{t}\left(\operatorname{obj}\left(\delta^{\prime}\right)\right)$ and $\mathbb{P}^{t}\left(\operatorname{sub}\left(\alpha, \chi, \delta^{\prime}\right)\right)$.

So how then will $\alpha$ specify: $\mathbb{P}^{t}(\operatorname{satisfy}(\alpha, \chi, \delta)), \mathbb{P}^{t}(\operatorname{sub}(\alpha, \chi, \delta))$ and $\mathbb{P}^{t}(\operatorname{obj}(\delta))$ ? Of these three factors only $\mathbb{P}^{t}(\operatorname{obj}(\delta))$ has a clear meaning, but it may only be estimated if there is sufficient market data available. In the case of selling sardines this may well be so, but in the case of Google launching a take-over bid for Microsoft it will $\operatorname{not}^{6}$. Concerning $\mathbb{P}^{t}(\operatorname{satisfy}(\alpha, \chi, \delta))$ and $\mathbb{P}^{t}(\operatorname{sub}(\alpha, \chi, \delta))$ we assume that an agent will somehow assess each of these as some combination of the confidence levels across a set of privately-known criteria. For example, if I am buying a camera then I may be prepared to define:

$$
\mathbb{P}^{t}(\operatorname{satisfy}(\alpha, \chi, \delta))=h\left(\mathbb{P}^{t}(\text { easy-to-use }(\alpha, \delta)), \mathbb{P}^{t}(\text { well-built }(\alpha, \delta))\right)
$$

for some function $h$. Any attempt to model another agent's $h$ function will be as difficult as modelling $g$ above. But, it is perfectly reasonable to suggest that by observing my argumentative dialogue an agent could form a view as to which of these two criteria above was more important.

This paper considers how an agent may observe the argumentative dialogue with the aim of modelling, within each of the three basic factors, the partner's criteria and the relative importance of those criteria. In repeated dealings between two agents, this model may be strengthened when the objects of the successive negotiations are semantically close but not necessarily identical.

\footnotetext{
${ }^{6}$ In this example the subjective valuation will be highly complex.
} 
John Debenham and Carles Sierra

\section{The Scenario}

Rhetorical argumentation is freed from the rigour of classical argumentation and descriptions of it can take the form of "this is how it works here" and "this is how it works there" without describing a formal basis. We attempt to improve on this level of vagary by using a general scenario and describing the behaviour of our agents within it.

In a general retail scenario there is a seller agent, $\alpha$, and a buyer, $\beta$. The items for sale are abstracted from: digital cameras, mobile phones, PDAs, smart video recorders, computer software, sewing machines and kitchen mixers. The features of an item are those that are typically listed on the last few pages of an instruction booklet. For example, a camera's features could include the various shutter speeds that it is capable of, the various aperture settings, the number of years of warranty, and so on - together the features describe the capabilities of the item. For the purpose of comparison with other items, $\beta$ will consider a particular item as a typed Boolean vector over the (possible) features of each item available, this vector shows which feature is present. The state of an item is then specified by identifying which of the item's features are 'on'. For example, the state of a camera could be: 'ready' with aperture set to ' $\mathrm{f} 8$ ' and shutter speed set to ' 1 500'th of a second'. In this scenario an offer is a pair (supply of a particular item, supply of some money) being $\alpha$ 's and $\beta$ 's commitments respectively.

$\beta$ may wish to know how well an item performs certain tasks. Software agents are not naturally endowed with the range of sensory and motor functions to enable such an evaluation. We imagine that the seller agent has an associated tame human who will demonstrate how the various items perform particular tasks on request, but performs no other function. We also imagine that the buyer agent has an associated tame human who can observe what is demonstrated, articulates an evaluation of it that is passed to its own agent, but performs no other function.

To simplify our set up we assume that the seller, $\alpha$, is $\beta$ 's only source of information about what tasks each item can perform, and, as we describe below, what sequence of actions are necessary to make an item perform certain tasks ${ }^{7}$. That is, our multiagent system consists only of $\{\alpha, \beta\}$, and the buyer is denied access to product reviews, but does have access to market pricing data. This restriction simplifies the interactions and focusses the discussion on the argumentation.

For example, if the item is a camera the buyer may wish to observe how to set the camera's states so that it may be used for 'point-and-shoot' photography. If the item is a sewing machine she may wish to see how to make a button hole on a piece of cloth. If the item is graphics software she may wish to see how to draw a polygon with a two-pixel red line and to colour the polygon's interior blue. These tasks will be achieved by enacting a process that causes the item to pass though a sequence of states that will be explained to $\beta$ by $\alpha$. So far our model consists of: features, states, sequences and tasks.

\footnotetext{
${ }^{7}$ In other words, the sort of information that is normally available in the item's Instruction Booklet - we assume that $\alpha$ conveys this information accurately.
} 
We assume that the object of the negotiation is clear where the object is an uninstantiated statement of what both agents jointly understand as the intended outcome - e.g. I wish to exchange a quantity of eggs of certain quality for cash. We assume that each agent is negotiating with the aim of satisfying some goal or need that is private knowledge. In determining whether a negotiation outcome is acceptable in satisfaction of a need we assume that an agent will blend the factors in our acceptance model described in Section 3. We assume that for each factor an agent will articulate a set of criteria that together determine whether the factor is acceptable. The criteria may include private information such as deadlines.

More formally, there is a set of feature names, $\mathscr{F}$, a set of item names, $\mathscr{I}$, a feature mapping: feature : $\mathscr{I} \rightarrow x^{n}(\mathbb{B}: \mathscr{F})$ where there are $n$ feature names, and $\mathbb{B}$ is a boolean variable that may be $T$ or $\perp$. Each item name belongs to a unique concept - e.g.: "Nikon123 is-a camera". For any particular item name, $v$, feature $(v)$ will be a typed Boolean vector indicating which features that item $v$ possesses. Let $\mathscr{F}_{v}$ be the set of $n_{v}$ features that item $v$ possesses. At any particular time $t$, the state of an item is a mapping: state ${ }^{t}: \mathscr{I} \rightarrow \times^{n_{v}}\left(\mathbb{B}: \mathscr{F}_{v}\right)$ where the value $\top$ denotes that the corresponding feature of that item is 'on'. A sequence is an ordered set of states, $\left(\mathbf{w}_{i}\right)$, where successive states differ in one feature only being on and off. A sequence is normally seen as performing a task that are linked by the mapping: to-do : $\mathscr{T} \rightarrow 2^{\mathscr{S}}$ where $\mathscr{T}$ is the set of tasks and $\mathscr{S}$ the set of all possible sequences - that is, there many be several sequences that perform a task. If a sequence is performed on an item then, with the assistance of a human, the agent rates how well it believes the sequence performs the associated task. The evaluation space, $\mathscr{E}$, could be $\{\operatorname{good}, \mathrm{OK}, \mathrm{bad}\}$. A criterion is a predicate: $\operatorname{criterion}(v)$, meaning that the item $v$ satisfies criterion 'criterion'. The set of criteria is $\mathscr{C}$.

\section{The Buyer Assesses A Contract}

In this Section we consider how the buyer might use the general framework in Section 3 to assess a contract ${ }^{8}$. In general an agent will be concerned about the enactment of any contract signed as described in Equation 1. In the scenario described in Section 4, enactment is not an issue, and so we focus on Equation 2. To simplify things we ignore the subjective valuation factor. Before addressing the remaining two factors we note that the buyer will not necessarily be preference aware.

First $\beta$ must give meaning to $\mathbb{P}^{t}(\operatorname{satisfy}(\beta, \chi, \delta))$ by defining suitable criteria and the way that the belief should be aggregated across those criteria. Suppose one of $\beta$ 's criteria is $\mathbb{P}^{t}$ (ease-of-use $(\beta, \delta)$ ). The idea is that $\beta$ will ask $\alpha$ to demonstrate how certain tasks are performed, will observe the sequences that $\alpha$ performs, and will use those observations to revise this probability distribution until some clear verdict appears.

\footnotetext{
${ }^{8}$ The seller will have little difficulty in deciding whether a contract is acceptable if he knows what the items cost.
} 
Suppose the information acquisition process is managed by a plan $\pi$. Let random variable $X$ represent $\mathbb{P}^{t}$ (ease-of-use $(\beta, \delta)=e_{i}$ ) where the $e_{i}$ are values from an evaluation space that could be $\mathscr{E}=\{$ fantastic, acceptable, just OK, shocking $\}$. Then given a sequence $s$ that was supposed to achieve task $\tau$, suppose that $\beta$ 's tame human rates $s$ as evidence for ease-of-use as $e \in \mathscr{E}$ with probability $z$. Suppose that $\beta$ attaches a weighting $\mathbb{R}^{t}(\pi, \tau, s)$ to $s, 0<\mathbb{R}<1$, which is $\beta$ 's estimate of the significance of the observation of sequence $s$ within plan $\pi$ as an indicator of the true value of $X$. For example, the on the basis of the observation alone $\beta$ might rate ease-of-use as $e=$ acceptable with probability $z=0.8$, and separately give a weighting of $\mathbb{R}^{t}(\pi, \tau, s)=0.9$ to the sequence $s$ as an indicator of ease-of-use. For an information-based agent each plan $\pi$ has associated update functions, $J_{\pi}(\cdot)$, such that $J_{\pi}^{X}(s)$ is a set of linear constraints on the posterior distribution for $X$. In this example, the posterior value of 'acceptable' would simply be constrained to 0.8 .

Denote the prior distribution $\mathbb{P}^{t}(X)$ by $\mathbf{p}$, and let $\mathbf{p}_{(s)}$ be the distribution with minimum relative entropy ${ }^{9}$ with respect to $\mathbf{p}: \mathbf{p}_{(s)}=\arg \min _{\mathbf{r}} \sum_{j} r_{j} \log \frac{r_{j}}{p_{j}}$ that satisfies the constraints $J_{s}^{X}(s)$. Then let $\mathbf{q}_{(s)}$ be the distribution:

$$
\mathbf{q}_{(s)}=\mathbb{R}^{t}(\pi, \tau, s) \times \mathbf{p}_{(s)}+\left(1-\mathbb{R}^{t}(\pi, \tau, s)\right) \times \mathbf{p}
$$

and then let:

$$
\mathbb{P}^{t}\left(X_{(s)}\right)= \begin{cases}\mathbf{q}_{(s)} & \text { if } \mathbf{q}_{(s)} \text { is more interesting than } \mathbf{p} \\ \mathbf{p} & \text { otherwise }\end{cases}
$$

A general measure of whether $\mathbf{q}_{(s)}$ is more interesting than $\mathbf{p}$ is: $\mathbb{K}\left(\mathbf{q}_{(s)} \| \mathbb{D}(X)\right)>$ $\mathbb{K}(\mathbf{p} \| \mathbb{D}(X))$, where $\mathbb{K}(\mathbf{x} \| \mathbf{y})=\sum_{j} x_{j} \log \frac{x_{j}}{y_{j}}$ is the Kullback-Leibler distance between two probability distributions $\mathbf{x}$ and $\mathbf{y}$, and $\mathbb{D}(X)$ is the expected distribution in the absence of any observations $-\mathbb{D}(X)$ could be the maximum entropy distribution. Finally, $\mathbb{P}^{t+1}(X)=\mathbb{P}^{t}\left(X_{(s)}\right)$. This procedure deals with integrity decay, and with two probabilities: first, the probability $z$ in the rating of the sequence $s$ that was intended to achieve $\tau$, and second $\beta$ 's weighting $\mathbb{R}^{t}(\pi, \tau, s)$ of the significance of $\tau$ as an indicator of the true value of $X$. Equation 5 is intended to prevent weak information from decreasing the certainty of $\mathbb{P}^{t+1}(X)$. For example if the current distribution is $(0.1,0.7,0.1,0.1)$, indicating an "acceptable" rating, then weak evidence $\mathbb{P}(X=$ acceptable $)=0.25$ is discarded .

Equation 4 simply adds in new evidence $\mathbf{p}_{(s)}$ to $\mathbf{p}$ weighted with $\mathbb{R}^{t}(\pi, \tau, s)$. This is fairly crude, but the observations are unlikely to be independent and the idea is

${ }^{9}$ Given a probability distribution $\mathbf{q}$, the minimum relative entropy distribution $\mathbf{p}=\left(p_{1}, \ldots, p_{I}\right)$ subject to a set of $J$ linear constraints $\mathbf{g}=\left\{g_{j}(\mathbf{p})=\mathbf{a}_{\mathbf{j}} \cdot \mathbf{p}-c_{j}=0\right\}, j=1, \ldots, J$ (that must include the constraint $\left.\sum_{i} p_{i}-1=0\right)$ is: $\mathbf{p}=\arg \min _{\mathbf{r}} \sum_{j} r_{j} \log \frac{r_{j}}{q_{j}}$. This may be calculated by introducing Lagrange multipliers $\lambda: L(\mathbf{p}, \lambda)=\sum_{j} p_{j} \log \frac{p_{j}}{q_{j}}+\lambda \cdot \mathbf{g}$. Minimising $L,\left\{\frac{\partial L}{\partial \lambda_{j}}=g_{j}(\mathbf{p})=0\right\}, j=1, \ldots, J$ is the set of given constraints $\mathbf{g}$, and a solution to $\frac{\partial L}{\partial p_{i}}=0, i=1, \ldots, I$ leads eventually to $\mathbf{p}$. Entropybased inference is a form of Bayesian inference that is convenient when the data is sparse [2] and encapsulates common-sense reasoning [7]. 
that $\pi$ will specify a "fairly comprehensive" set of tasks aimed to determine $\mathbb{P}^{t}(X)$ to a level of certainty sufficient for Equation 2.

$\mathbb{P}^{t}(\operatorname{obj}(\boldsymbol{\delta}))$ estimates the belief that $\delta$ is acceptable in the open-market that $\beta$ may observe in the scenario. Information-based agents model what they don't know with certainty as probability distributions. Suppose that $X$ is a discrete random variable whose true value is the open-market value of an item. First, $\beta$ should be able to bound $X$ to an interval $\left(x_{\min }, x_{\max }\right)$ - if this is all the evidence that $\beta$ can muster then $X$ will be the flat distribution (with maximum entropy) in this interval, and $\mathbb{P}^{t}\left(\operatorname{obj}((\right.$ item,$y))=\sum_{x>y} \mathbb{P}(X=x)$. $\beta$ may observe evidence, perhaps as observed sale prices for similar items, that enables him to revise particular values in the distribution for $X$. A method [9] similar to that described above is used to derive the posterior distribution - it is not detailed here. An interesting aspect of this approach is that it works equally well when the valuation space has more than one dimension.

\section{The Seller Models the Buyer}

In this Section we consider how the seller might model the buyer's contract acceptance logic in an argumentative context. As in Section 5 we focus on Equation 2 and for reasons of economy concentrate on the factor: $\mathbb{P}^{t}(\operatorname{satisfy}(\alpha, \chi, \delta))$.

Suppose that $\beta$ has found an item that he wants to buy, $\alpha$ will be interested in how much he is prepared to pay. In a similar way to Section $5, \alpha$ can interpret $\beta$ 's proposals as willingness to accept the offers proposed, and counter-offers as reluctance to accept the agent's prior offer - all of these interpretations being qualified with an epistemic belief probability. Entropy-based inference is then used to derive a complete probability distribution over the space of offers for a random variable that represents the partner's limit offers. This distribution is "the least biased estimate possible on the given information; i.e. it is maximally noncommittal with regard to missing information" [3]. If there are $n$-issues then the space of limit offers will be an $(n-1)$-dimensional surface through offer space.

$\alpha$ 's world model, $\mathscr{M}^{t}$, contains probability distributions that model the agent's belief in the world, including the state of $\beta$. In particular, for every criterion $c \in \mathscr{C}$ $\alpha$ associates a random variable $C$ with probability mass function $\mathbb{P}^{t}\left(C=e_{i}\right)$.

The distributions that relate object to criteria may be learned from prior experience. If $\mathbb{P}^{t}(C=e \mid O=o)$ is the prior distribution for criteria $C$ over an evaluation space given that the object is $o$, then given evidence from a completed negotiation with object $o$ we use the standard update procedure described in Section 5. For example, given evidence that $\alpha$ believes with probability $p$ that $C=e_{i}$ in a negotiation with object $o$ then $\mathbb{P}^{t+1}(C=e \mid O=o)$ is the result of applying the constraint $\mathbb{P}\left(C=e_{i} \mid O=o\right)=p$ with minimum relative entropy inference as described previously, where the result of the process is protected by Equation 5 to ensure that weak evidence does not override prior estimates.

In the absence of evidence of the form described above, the distributions, $\mathbb{P}^{t}(C=$ $e \mid O=o$ ), should gradually tend to ignorance. If a decay-limit distribution [9] is 
known they should tend to it otherwise they should tend to the maximum entropy distribution.

In a multiagent system, this approach can be strengthened in repeated negotiations by including the agent's identity, $\mathbb{P}^{t}(C=e \mid(O=o$, Agent $=\beta))$ and exploiting a similarity measure across the ontology. So if $\beta$ purchased a kitchen mixer apparently with the criterion "easy to carry" then that would increase the prior probability that $\beta$ will use the criterion "easy to carry" in negotiating for a sewing machine. Two methods for propagating estimates across the world model by exploiting the $\operatorname{Sim}(\cdot)$ measure are described in [9]. An extension of the $\operatorname{Sim}(\cdot)$ measure to sets of concepts is straightforward, we will note it as $\operatorname{Sim} *(\cdot)$.

Agent $\beta$ 's disposition is the underlying rationale that he has for a dialogue. $\alpha$ will be concerned with the confidence in $\alpha$ 's beliefs of $\beta$ 's disposition as this will affect the certainty with which $\alpha$ believes she knows $\beta$ 's key criteria. Gauging disposition in human discourse is not easy, but is certainly not impossible. We form expectations about what will be said next; when those expectations are challenged we may well believe that there is a shift in the rationale.

The bargaining literature consistently advises (see for example [5]) that an agent should change its stance (one dimension of stance being the 'nice guy' / 'tough guy' axis) to prevent other agents from decrypting their private information, and so we should expect some sort of "smoke screen" surrounding any dialogue between competitive agents. It would be convenient to think of disposition as the mirror-image of stance, but what matters is the agent's confidence in its model of the partner. The problem is to differentiate between a partner that is skilfully preventing us from decrypting their private information, and a partner that has either had a fundamental change of heart or has changed his mind in a way that will significantly influence the set of contracts that he will agree to. The first of these is normal behaviour, and the second means that the models of the partner may well be inaccurate.

$\alpha$ 's model of $\beta$ 's disposition is $D_{C}=\mathbb{P}^{t}(C=e \mid O=o)$ for every criterion in the ontology, where $o$ is the object of the negotiation. $\alpha$ 's confidence in $\beta$ 's disposition is the confidence he has in these distributions. Given a negotiation object $o$, confidence will be aggregated from $\mathbb{H}(C=e \mid O=o)$ for every criterion in the ontology. Then the idea is that if in the negotiation for a camera "for family use" $\alpha$ is asked to demonstrate how to photograph a drop of water falling from a tap then this would presumably cause a dramatic difference between $\mathbb{P}^{t}(C=e \mid(O=$ "family use" $))$ and $\mathbb{P}^{t}\left(C=e \mid\left(O=\right.\right.$ "family use", $O^{\prime}=$ "photograph water drops" $\left.)\right)$. This difference causes $\alpha$ to revise her belief in "family use", to revise the disposition towards distributions of higher entropy, and to approach the negotiation on a broader basis.

\section{Strategies}

In this section we describe the components of an argumentation strategy starting with tools for valuing information revelation that are used to model the fairness of a negotiation dialogue. 
Everything that an agent communicates gives away information. The simple offer "you may purchase this wine for €3" may be intrepretd in a utilitarian sense (e.g. the profit that you could make by purchasing it), and as information (in terms of the reduction of your entropy or uncertainty in your beliefs about my limit price for the item). Information-based agents value information exchanged, and attempt to manage the associated costs and benefits.

Illocutionary categories and an ontology together form a framework in which the value of information exchanged can be categorised. The LOGIC framework for argumentative negotiation [11] is based on five illocutionary categories: Legitimacy of the arguments, Options i.e. deals that are acceptable, Goals i.e. motivation for

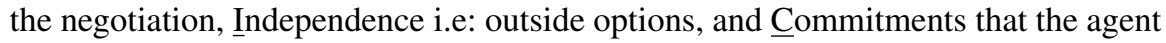
has including its assets. In general, $\alpha$ has a set of illocutionary categories $\mathscr{Y}$ and a categorising function $\kappa: \mathscr{L} \rightarrow \mathscr{P}(\mathscr{Y})$. The power set, $\mathscr{P}(\mathscr{Y})$, is required as some utterances belong to multiple categories. For example, in the LOGIC framework the utterance "I will not pay more for a bottle of Beaujolais than the price that John charges" is categorised as both Option (what I will accept) and Independence (what I will do if this negotiation fails).

Then two central concepts describe relationships and dialogues between a pair of agents. These are intimacy - degree of closeness, and balance - degree of fairness. In this general model, the intimacy of $\alpha$ 's relationship with $\beta, A^{t}$, measures the amount that $\alpha$ knows about $\beta$ 's private information and is represented as real numeric values over $\mathscr{G}=\mathscr{Y} \times V$.

Suppose $\alpha$ receives utterance $u$ from $\beta$ and that category $y \in \kappa(u)$. For any concept $x \in V$, define $\Delta(u, x)=\max _{x^{\prime} \in \operatorname{concepts}(u)} \operatorname{Sim}\left(x^{\prime}, x\right)$. Denote the value of $A_{i}^{t}$ in position $(y, x)$ by $A_{(y, x)}^{t}$ then:

$$
A_{(y, x)}^{t}=\rho \times A_{(y, x)}^{t-1}+(1-\rho) \times \mathbb{I}(u) \times \Delta(u, x)
$$

for any $x$, where $\rho$ is the discount rate, and $\mathbb{I}(u)$ is the information ${ }^{10}$ in $u$. The balance of $\alpha$ 's relationship with $\beta_{i}, B^{t}$, is the element by element numeric difference of $A^{t}$ and $\alpha$ 's estimate of $\beta$ 's intimacy on $\alpha$.

We are particularly interested in the concept of intimacy in so far as it estimates what $\alpha$ knows about $\beta$ 's criteria, and about the certainty of $\alpha$ 's estimates of the random variables $\left\{C_{i}\right\}$. We are interested in balance as a measure of the 'fairness' of the dialogue. If $\alpha$ shows $\beta$ how to take a perfect photograph of a duck then it is reasonable to expect some information at least in return.

Moreover, $\alpha$ acts proactively to satisfy her needs - that are organised in a hierarchy ${ }^{11}$ of needs, $\Xi$, and a function $\omega: \Xi \rightarrow \mathscr{P}(W)$ where $W$ is the set of perceivable states, and $\omega(\chi)$ is the set of states that satisfy need $\chi \in \Xi$. Needs turn 'on' spontaneously, and in response to triggers. They turn 'off' because $\alpha$ believes they are

\footnotetext{
${ }^{10}$ Information is measured in the Shannon sense, if at time $t, \alpha$ receives an utterance $u$ that may alter this world model then the (Shannon) information in $u$ with respect to the distributions in $\mathscr{M}^{t}$ is: $\mathbb{I}(u)=\mathbb{H}\left(\mathscr{M}^{t}\right)-\mathbb{H}\left(\mathscr{M}^{t+1}\right)$.

${ }^{11}$ In the sense of the well-known Maslow hierarchy [6], where the satisfaction of needs that are lower in the hierarchy take precedence over the satisfaction of needs that are higher.
} 
satisfied. When a need fires, a plan is chosen to satisfy that need (we do not describe plans here). If $\alpha$ is to contemplate the future she will need some idea of her future needs — this is represented in her needs model: $v: T \rightarrow x^{|\Xi|}[0,1]$ where $T$ is time, and: $v(t)=\left(\chi_{1}^{t}, \ldots, \chi_{|\Xi|}^{t}\right)$ where $\chi_{i}^{t}=\mathbb{P}\left(\right.$ need $\chi_{i}$ fires at time $\left.t\right)$.

Given the needs model, $v, \alpha$ 's relationship model (Relate $(\cdot))$ determines the target intimacy, $A_{i}^{* t}$, and target balance, $B_{i}^{* t}$, for each agent $i$ in the known set of agents Agents. That is, $\left\{\left(A_{i}^{* t}, B_{* i}^{t}\right)\right\}_{i=1}^{\mid \text {Agents } \mid}=\operatorname{Relate}(v, \mathbf{X}, \mathbf{Y}, \mathbf{Z})$ where, $\mathbf{X}_{i}$ is the trust model, $\mathbf{Y}_{i}$ is the honour model and $\mathbf{Z}_{i}$ is the reliability model as described in [9]. As noted before, the values for intimacy and balance are not simple numbers but are structured sets of values over $\mathscr{Y} \times V$.

When a need fires $\alpha$ first selects an agent $\beta_{i}$ to negotiate with - the social model of trust, honour and reliability provide input to this decision, i.e. $\beta_{i}=$ $\operatorname{Select}(\chi, \mathbf{X}, \mathbf{Y}, \mathbf{Z})$. We assume that in her social model, $\alpha$ has medium-term intentions for the state of the relationship that she desires with each of the available agents - these intentions are represented as the target intimacy, $A_{i}^{* t}$, and target balance, $B_{i}^{* t}$, for each agent $\beta_{i}$. These medium-term intentions are then distilled into short-term targets for the intimacy, $A_{i}^{* * t}$, and balance, $B_{i}^{* * t}$, to be achieved in the current dialogue $\Psi^{t}$, i.e. $\left(A_{i}^{* * t}, B_{i}^{* * t}\right)=\operatorname{Set}\left(\chi, A_{i}^{* t}, B_{i}^{* t}\right)$. In particular, if the balance target, $B_{i}^{* * t}$, is grossly exceeded by $\beta$ failing to co-operate then it becomes a trigger for $\alpha$ to terminate the negotiation.

For an information-based agent, an incoming utterance is only of interest if it reduces the uncertainty (entropy) of the world model in some way. In informationbased argumentation we are particularly interested in the effect that an argumentative utterance has in the world model including $\beta$ 's disposition, and $\alpha$ 's estimate of $\beta$ 's assessment of current proposals in terms of its criteria.

Information-based argumentation attempts to counter the effect of the partner's arguments, in the simple negotiation protocol used here, an argumentative utterance, $u$, will either contain a justification of the proposal it accompanies, a rating and justification of one of $\alpha$ demonstration sequences, or a counter-justification of one of $\alpha$ 's prior proposals or arguments. If $u$ requests $\alpha$ to perform a task then $u$ may modify $\beta$ 's disposition i.e. the set of conditional estimates of the form: $\mathbb{P}^{t}(C=e \mid O=$ $o)$ ). If $\beta$ rates and comments on the demonstration of a sequence then this affects $\alpha$ 's estimate of $\beta$ 's likelihood to accept a contract as described in Equation 1 (this is concerned with how $\beta$ will apply his criteria).

Suppose that $u$ rates and comments on the performance of a sequence then that sequence will have been demonstrated in response to a request to perform a task. Given a task, $\tau$, and a object, $s, \alpha$ may have estimates for $P^{t}(C=e \mid(O=o, \mathscr{T}=\tau))$ - if so then this suggests a link between the task and a set of one or more criteria $C_{u}$. The effect that $u$ has on $\beta$ 's criteria (what ever they are) will be conveyed as the rating. In the spirit of the scenario, we assume that for every criterion and object pair $(C, o) \alpha$ has a supply of positive argumentative statements $\mathscr{L}_{(C, o)}$. Suppose $\alpha$ wishes to counter the negatively rated $u$ with a positively rated $u^{\prime}$. Let $\Psi_{u}$ be the set of all arguments exchanged between $\alpha$ and $\beta$ prior to $u$ in the dialogue. Let $M_{u} \subseteq \mathscr{L}_{(C, o)}$ for any $C \in C_{\mu}$. Let $N_{u} \subseteq M_{u}$ such that $\forall x \in N_{u}$ and $\forall u^{\prime} \in \Psi_{u}$, $\operatorname{Sim} *\left(\operatorname{concepts}(x)\right.$, concepts $\left.\left(u^{\prime}\right)\right)>\eta$ for some constant $\eta$. So $N_{u}$ is a set of argu- 
ments all of which (a) have a positive effect on at least one criterion associated with the negative $u$, and (b) are at 'some distance' (determined by $r$ ) from arguments already exchanged. Then:

$$
u^{\prime}= \begin{cases}\arg \min _{u^{\prime} \in N_{u}} \operatorname{Sim} *\left(\operatorname{concepts}(u), \text { concepts }\left(u^{\prime}\right)\right) & \text { if } N_{u} \neq \emptyset \\ \arg \min _{u^{\prime} \in M_{u}} \operatorname{Sim} *\left(\text { concepts }(u), \text { concepts }\left(u^{\prime}\right)\right) & \text { otherwise. }\end{cases}
$$

So using only 'fresh' arguments, $\alpha$ prefers to choose a counter argument to $u$ that is semantically close to $u$, and if that is not possible she chooses an argument that has some general positive effect on the criteria and may not have been used previously.

Suppose that $u$ proposes a contract. $\alpha$ will either decide to accept it or to make a counter offer. We do not describe the bargaining process here, see [9].

\section{Discussion}

If $\beta_{i}$ communicates $u$ then $\alpha$ responds with:

$$
\left.u^{\prime}=\operatorname{Argue}\left(u, \mathscr{M}^{t}, \Psi^{t}, A^{* * t}, B^{* * t}, C_{u}, N_{u}, M_{u}, D_{u}\right)\right)
$$

where:

- the negotiation mechanisms as explained in Section 7 sets parameters $A^{* * t}, B^{* * t}$ ) (see e.g. [11] for further details);

- the argumentation process determines the parameters $N_{u}, M_{u}$ needed to generate the accompanying arguments to the proposal, see Section 7;

- the criteria modeling process determines the set of criteria $C_{u}$ used by our opponent to assess the proposals, see Section 6; and,

- the disposition modeling sets the distributions $D_{u}$ used to interpret the stance of the opponent, see Section 6.

We have described an approach to argumentation that aims to:

- discover what the partner's key evaluative criteria are,

- model how the partner is evaluating his key criteria given some evidence,

- influence the partner's evaluation of his key criteria,

- influence the relative importance that the partner attaches to those criteria, and

- introduce new key criteria when it is strategic to do so.

The ideas described here are an attempt to develop an approach to argumentation that may be used in the interests of both parties. It aims to achieve this by unearthing the 'top layer' of the partner's reasoning apparatus and by attempting to work with it rather than against it. To this end, the utterances produced aim to influence the partner to believe what we believe to be in his best interests - although it may not be in fact. The utterances aim to convey what is so, and not to point out "where the partner is wrong". In the long term, this behaviour is intended to lead to the 
development of lasting relationships between agents that are underpinned both by the knowledge that their partners "treat them well" and that their partners act as they do "for the right reasons".

In previous work [11] we have advocated the gradual development of trust and intimacy $^{12}$ through successive argumentative exchanges as a way of building relationships between agents. In this paper we have gone one step further by including a modest degree of understanding in the sense that an agent attempts to understand what her partner likes. This augments the tools for building social relationships through argumentation by establishing:

- trust - my belief in the veracity of your commitments

- intimacy - my belief in the extent to which I know your private information

- understanding - my belief in the extent to which I know what you like

Acknowledgements This research has been supported by the Sabbatical Programme of the Catalan Government BE2007, the Australian Research Council Discovery Grant DP0557168, and by the Spanish Ministerio de Educación y Ciencia project "Agreement Technologies" (CONSOLIDER CSD2007-0022, INGENIO 2010).

\section{References}

1. Arcos, J.L., Esteva, M., Noriega, P., Rodríguez, J.A., Sierra, C.: Environment engineering for multiagent systems. Journal on Engineering Applications of Artificial Intelligence 18 (2005)

2. Cheeseman, P., Stutz, J.: Bayesian Inference and Maximum Entropy Methods in Science and Engineering, chap. On The Relationship between Bayesian and Maximum Entropy Inference, pp. 445 - 461. American Institute of Physics, Melville, NY, USA (2004)

3. Jaynes, E.: Information theory and statistical mechanics: Part I. Physical Review 106, 620 630 (1957)

4. Kalfoglou, Y., Schorlemmer, M.: IF-Map: An ontology-mapping method based on information-flow theory. In: S. Spaccapietra, S. March, K. Aberer (eds.) Journal on Data Semantics I, Lecture Notes in Computer Science, vol. 2800, pp. 98-127. Springer-Verlag: Heidelberg, Germany (2003)

5. Lewicki, R.J., Saunders, D.M., Minton, J.W.: Essentials of Negotiation. McGraw Hill (2001)

6. Maslow, A.H.: A theory of human motivation. Psychological Review 50, 370-396 (1943)

7. Paris, J.: Common sense and maximum entropy. Synthese 117(1), 75 - 93 (1999)

8. Rahwan, I., Ramchurn, S., Jennings, N., McBurney, P., Parsons, S., Sonenberg, E.: Argumentation-based negotiation. Knowledge Engineering Review 18(4), 343-375 (2003)

9. Sierra, C., Debenham, J.: Trust and honour in information-based agency. In: P. Stone, G. Weiss (eds.) Proceedings Fifth International Conference on Autonomous Agents and Multi Agent Systems AAMAS-2006, pp. 1225 - 1232. ACM Press, New York, Hakodate, Japan (2006)

10. Sierra, C., Debenham, J.: Information-based agency. In: Proceedings of Twentieth International Joint Conference on Artificial Intelligence IJCAI-07, pp. 1513-1518. Hyderabad, India (2007)

11. Sierra, C., Debenham, J.: The LOGIC Negotiation Model. In: Proceedings Sixth International Conference on Autonomous Agents and Multi Agent Systems AAMAS-2007, pp. 1026-1033. Honolulu, Hawai'i (2007)

\footnotetext{
12 The revelation of private information.
} 